\title{
Advancing women in R\&D will help accelerate medicine
}

\author{
Annalisa Jenkins
}

\begin{abstract}
Enabling women to serve at the highest leadership levels in pharmaceutical R\&D will help advance science to offer a broader array of medicines to patients. But in an industry dominated by men in the most seniorlevel roles, women have a long way to go to get to the top.
\end{abstract}

In the US, women make $80 \%$ of the healthcare choices for their families ${ }^{1}$. Paradoxically, however, none of the R\&D leaders among the world's top 15 pharmaceutical companies is a woman. The absence of women in the ranks of the senior leaders who ultimately make the decisions that determine consumers' healthcare options represents a major challenge, and we must work to address it.

Enabling women to serve at the highest leadership levels in biopharma R\&D will do a great deal of good. For example, substantially expanding the industry's talent pool will improve the pace, scope and quality of scientific and medical innovation. Moreover, it will finally put women's unique perspectives on healthcare issues, needs and decisions to use-particularly in the increasingly important area of fertility.

This past September, when I was appointed the Global Head of R\&D for Merck Serono (a German company known as EMD Serono in North America), I became the only woman in this role across the 20 largest biopharmaceutical companies in the world. As part of my mission at the company, I hope to develop not only innovative new medicines in the areas of oncology, multiple sclerosis and infertility but also new opportunities for development for the women with whom I work.

Why do so few women lead the R\&D divisions of other major pharmaceutical companies? Certainly, gender favoritism plays a part in this paradox in an industry dominated by men. But another factor also has a lead role: whereas most men succeed at having both a career and a family, women more commonly face the challenge of having to choose one or the other.

In the world of academic science and medicine, women who choose to delay starting a family in order to build a career in health care and research may be putting their fertility at risk. For example, a woman is typically at least 30 years old when she achieves the rank of an assistant professor. This means that she will not be eligible for tenure until she is at least 35 or 36 at the earliest. So, in the prime of her career-precisely when years of demanding and dedicated work mean that she can achieve a strong professional reputation in science and thereby do more innovative, independent and influential work-she is also at the peak of her reproductive health.

When women in research start a family, they often shoulder the majority of child-rearing tasks in addition to their responsibilities in the lab or hospital. This, of course, puts them at a disadvantage in relation to their colleagues and peers who have only one major responsibility, not two. Perhaps it's not surprising, then, that $21 \%$ of women scientists and engineers say that balancing family life and work life is a career obstacle, but only $4.5 \%$ of men say the same thing ${ }^{2}$.

Indeed, in the fields of science, medicine and biopharmaceutical $\mathrm{R} \& \mathrm{D}$, it's particularly difficult to put professional activities on hold in order to have babies and raise young children. Scientific and medical knowledge is expanding so rapidly that being professionally inactive for even a relatively short time can have costly consequences for a person's career.
Ultimately, overcoming the imbalance between women and men in leadership roles in the pharmaceutical industry will require practical support systems-for example, in the form of better leave policies for new mothers and fathers and more on-site childcare. At Merck Serono, we have just instituted a program called Work at Merck that allows for telecommuting and time off for family care. In the same vein, the brand-new headquarters for Biogen Idec in Cambridge, Massachusetts, includes an 8,000-square-foot daycare center.

Flexibility in work schedules is important. In an interview with the website Pharmafile.com earlier this year, Jane Griffiths, the first female company group chairman of Johnson \& Johnson's Janssen pharma unit in Europe, the Middle East and Africa, cited flexible work hours and the ability to work from home as two ways in which drug companies can help support working women.

I have two children, whom I cherish. As young adults, they are starting to find their way in the world. My pride in them-which is partly based on their efforts to use education and health care to help other people-is immeasurable. My daughter is a biology major in college and is already showing keen interest in making a difference in the world through volunteer efforts to help young girls further their studies in Africa. But my own journey as a parent, a physician and an executive has not been easy. I have had to use a complex mixture of hard work, excellent sponsorship by colleagues and exceptional energy to have my career and my children, too. (It's not surprising that, by far, the largest part of the mixture has been-and remains-work.)

Fortunately, I love the work I do. And, even more fortunately, I've been able to balance work and family life as a result of a strong network of personal and professional support. But I've had to make tough decisions and sacrifices that men in a similar position would not have to make, including leaving my position as a surgeon in the British Royal Navy because it was not possible at the time to have both a military medical career and a young family.

An important part of my job as head of R\&D at Merck Serono is to envision the future of the biopharmaceutical industry realistically yet optimistically. I believe and I certainly hope that as our industry grows in importance and impact the professional opportunities available to women will expand exponentially. At the same time, I predict the culture of biopharma will change to reflect the value to everyone of balance. So today this is the imperative: to provide the pathways of learning, to open the doors to senior leadership roles, and to give women both an equal chance to build great careers in biopharma and the right support to be become new mothers and good parents.

1. United States Department of Labor. General facts on women and job based health. http://www.dol.gov/ebsa/newsroom/fshlth5.html (2013).

2. Preston, A. Are Women Achieving Equity in Chemistry? 129-148 (American Chemical Society, Washington, DC, 2006).

Annalisa Jenkins is the Global Head of Research \& Development at Merck Serono based in Darmstadt, Germany. 